\title{
TABLE ALPHABÉTIQUE DES
}

MATIĖRES CONTENUES DANS LES TROIS VOLUMES DES

\section{TABLEAUX DE LA RÉVOLUTION FRANÇAISE} PCBLIÉs

SUR LES PAPIERS INÉDITS

DU DEPARTEMENT ET DB LA POLICR SECRETR DE PARIS

PAR

ADOLPHE SCHMIDT,

rROFESSEUR D'RtGTOTRE A L'UNIVERSITÉ DE $\lambda$ ḰNA

1.EIPZIG,

VEIT \& COMP.

1871. 



\section{TABLE ALPHABÉTIQUE \\ IH:X}

MATIERES CONTENER IOANS I,FN TROJS VOLUMES

1, t:S

TABLEAUX DE LA RÉVOLUTION FRANÇAISE. 
